\title{
An investigation into the feasibility of an adaptive coaching smartphone application used in conjunction with a novel exercise programme in sedentary individuals with type 2 diabetes mellitus
}

\author{
Hugh Byrne \\ The Insight Centre for Data Analytics, \\ University College Dublin, Ireland \\ Hugh.byrne@insight-centre.org
}

Chris J. Thompson

Beaumont Hospital, Dublin

christhompson@beaumont.ie

\author{
Brian Caulfield \\ The Insight Centre for Data Analytics, \\ University College Dublin, Ireland \\ B.Caulfield@ucd.ie \\ Diarmuid Smith \\ Beaumont Hospital, Dublin \\ diarmuidsmith@beaumont.ie \\ Giuseppe De Vito \\ The Insight Centre for Data Analytics, \\ University College Dublin, Ireland \\ Giuseppe.devito@ucd.ie
}

\author{
Madeleine Lowery \\ The Insight Centre for Data Analytics, \\ University College Dublin, Ireland \\ madeleine.lowery@ucd.ie
}

\author{
Margaret Griffin \\ Beacon Hospital, Dublin \\ Marge101@eircom.net
}

\begin{abstract}
High levels of physical activity are of major importance in ensuring individuals live a healthy life and age successfully with limited impairments to their health status, function or overall quality of life. However, physical activity continues to be low worldwide, and rates of diseases and conditions associated with sedentary lifestyles are increasing. Furthermore, adherence to exercise prescriptions in individuals whose diseases or conditions are managed more effectively with high levels of physical activity is poor. This paper examines the feasibility of a novel adaptive coaching smartphone application in yielding a high adherence to a specific exercise programme, also designed to improve physical activity habits of previously sedentary individuals with type 2 diabetes. The findings of the current study suggest that combining the progressive short bout exercise programme with a novel adaptive coaching smartphone application is a feasible intervention in sedentary individuals with type 2 diabetes mellitus. The findings also suggest that the combined intervention can yield high adherences, increase overall physical activity levels and improve health related outcomes.
\end{abstract}

Categories and Subject Descriptors J.3 [Computer Applications]: Life and Medical Sciences, Healthcare information systems, Health informatics, Human computer interaction

Produces the permission block, and copyright information

†The full version of the author's guide is available as acmart.pdf document ${ }^{1}$ It is a datatype.

\section{Author Keywords}

Adaptive coaching; Monitoring; Exercise; Type 2 diabetes mellitus; Aging individuals.

\section{INTRODUCTION}

Physical activity plays a vital role in preventing or delaying the occurrence of many chronic diseases [1]. Similarly, it is a key element in the treatment of many chronic diseases, including type 2 diabetes mellitus (T2DM) [2, 3]. Nonetheless, adherence to physical activity remains to be low in chronic disease populations. Recommendations provided regarding levels of physical activity, for example achieving 30-60 minutes of moderate intensity activity on 5 days per week, are consistently ignored and new methods of improving compliance to physical activity are required [4]. Recent evidence suggests that short bouts of independently conducted walking or step training spread throughout the day can yield high adherences and improve health related outcomes in different populations, including individuals with type 2 diabetes. Similarly, progressive exercise programmes have been seen to have the potential to encourage motivation over long periods.

Recent studies have also highlighted that smartphone based coaching applications can improve health related outcomes in individuals with type 2 diabetes mellitus [5]. Automated coaching systems have also been shown to provide a cheap, private and convenient method of providing feedback to patients remotely. Furthermore, evidence suggests that computer based adaptive coaching systems can increase adherence to exercise programs [6]. These systems use theories of behavioural change to inform the automatic feedback provided to individuals to increase or maintain their motivation towards an exercise program and adjust the exercise prescription provided accordingly. The algorithms 
can regularly alter the level of physical activity prescribed to individuals based on their previous success to maintain motivation and adherence levels.

It seems plausible that a combination of novel approaches to exercise prescription and adaptive coaching strategies, delivered within the framework of a smartphone application, could deliver a meaningful impact in the management of diabetes. However, to date, the feasibility of implementing the exact combination of these two elements using a smartphone application has not been thoroughly evaluated across many populations, including in T2DM. This study reports on the feasibility of this combination being used over a 16-week period in previously sedentary individuals with T2DM. It will report on the adherence of the individuals to the programme and on the effects the intervention had on health-related outcome measures.

\section{METHODS}

\subsection{Participants}

7 individuals with type 2 diabetes for greater than 6 months (4 males, 3 females, $48.7 \pm 5.5$ years, BMI: $31.0 \pm 2.8$ ) were recruited through diabetes outpatients clinics in one public and one private hospital in Dublin, Ireland. They were all undertaking less than 150 minutes per week of physical activity, not treated with insulin, and had a HbA1c of 53$86 \mathrm{mmol} / \mathrm{mol}$. Individuals who had any mental, neurological, cardiovascular, musculoskeletal or orthopaedic deficiency contra-indicating regular training and/or muscular testing or making it impossible or preventing knowing consent to the study were not included. Similarly, individuals with current infectious or inflammatory condition, evidence of chronic renal failure or liver disease, severe proliferative diabetic retinopathy, current or recent pregnancy were excluded. The University Human Research Ethics Committee approved the study protocol.

\subsection{Study Protocol}

The exercise programme consisted of small bouts of walking and/or step training spread throughout the day to provide participants with greater autonomy and to remove Irish weather as a potential barrier to exercise. Sessions gradually increased in length, frequency and intensity and participants were asked to incorporate the exercise programme into their routines over 16 weeks. It was designed to encourage compliance and improve outcomes in line with recent evidence. The protocol started with 2-minute sessions twice/day at 12/20 on the Borg RPE scale on 3 days for the first week and progressed gradually in terms of duration, frequency and intensity to three 12-minute sessions each day at 16 on Borg scale in week 16.

\subsection{The adaptive coaching application}

The "exercise and coaching application group" downloaded the adaptive coaching application onto their smartphone at baseline. This application automatically provided daily and weekly reminders and encouragement to complete prescribed exercise sessions, record recent sessions and outlined the exercise session to be completed over the coming week. These messages included: "Congratulations! You completed all of your last exercise prescription. Can you complete all of these sessions today?" and "Congratulations, you completed X\% of exercise sessions in level Y. You are now progressing to level Z! Good luck!” amongst others.

Participants also used the application to record their adherence alongside a logbook. A function to record extra exercise sessions was also available in the application (see figure 1). The upcoming exercise prescriptions were adapted by the application based on this information input by the individual participants. The application treated each week as a "level" and participants needed to complete $75 \%$ of sessions within an individual week to progress onto the next. If they failed to complete $75 \%$ of sessions, they remained on that level the following week. This smart, adaptive coaching used a combination of the "goal setting" and "self-efficacy" theories of behavioural change to find an exercise prescription that best encouraged participants to alter their behaviour and motivation towards the progressive exercise prescription by ensuring the programme was not too hard and not too easy [6]. It automatically alters the weekly exercise prescription given to individual participants based on their adherence the previous week and adjusts the daily and weekly feedback messages provided the individual in line with this.

Figure 1 A screenshot taken from the application showing the day's prescription, the recording function along with the extra session function in the bottom right

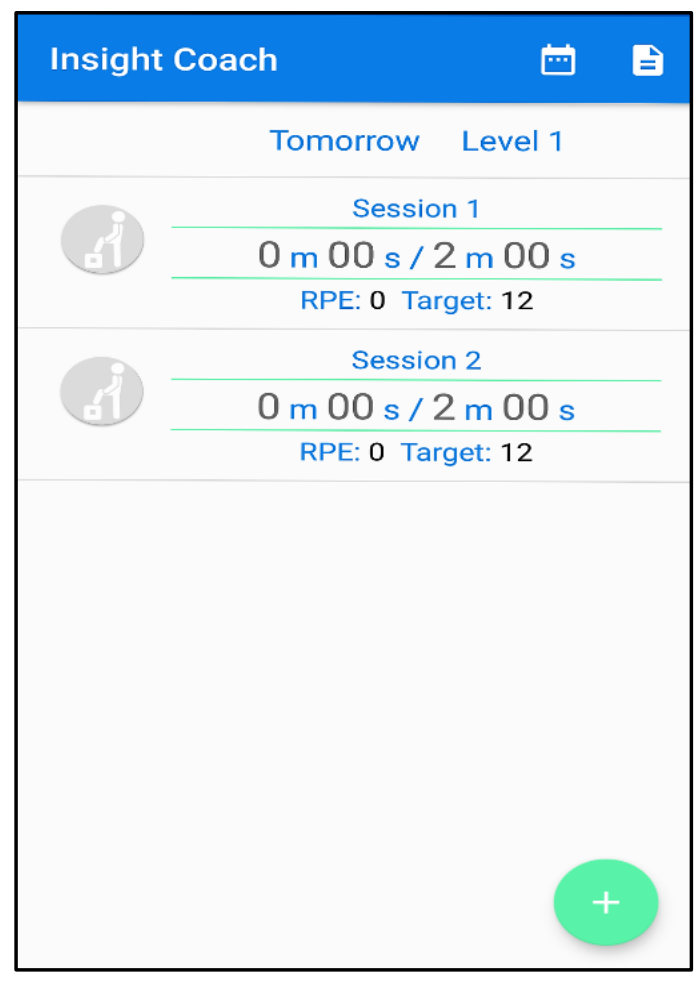




\subsection{Testing Protocol}

Following recruitment, all participants attended laboratory facilities at University College Dublin once before and once after the 16-week intervention period.

The testing protocol was outlined to participants when they arrived at the laboratory. Following this, they completed a consent from, Physical Activity Readiness Questionnaire and Active Australia Survey. Their height and weight were also recorded and a six-minute walk test was conducted. Blood samples were taken and analysed in their diabetes clinics. This procedure was repeated at all testing sessions.

Data is presented as means \pm standard deviation. All data was input into Microsoft Excel for analysis. Paired t-tests assuming equal variance were used to assess within group changes from baseline to follow up. A P value of $\leq 0.05$ was considered statistically significant.

\section{RESULTS}

Table 1 Baseline characteristics and dropouts

\begin{tabular}{|ll|}
\hline$(\mathrm{N}=7)$ & 3 \\
\hline Female & $2(1$ female $)$ \\
\hline Dropouts & $48.7 \pm 5.5$ \\
\hline Age (years) & $31.0 \pm 2.8$ \\
\hline BMI $(\mathrm{kg} / \mathrm{m} 2)$ & $5.3 \pm 1.6$ \\
\hline Disease Duration (years) & $63.8 \pm 4.7$ \\
\hline HbA1C (mmol/mol) & $72.9 \pm 45.6$ \\
\hline Activity minutes/week & \\
\hline
\end{tabular}

Baseline characteristics of participants and dropout rates can be seen in table 1 . Overall $2 / 7$ (28.6\%) failed to complete the intervention. The reasons for dropping out were sudden onset of knee pain and an unknown reason. One individual was unable to complete the study as planned due to a series of personal events that occurred at week 8 in the intervention period. This individual was included in the analysis.

Table 2 presents mean pre- and post-intervention data for those that completed the intervention period. $\mathrm{P}$ values presented are for within group differences from baseline. Some improvements in the health-related outcome measures were observed. Overall, adherences to prescribed exercise sessions was high. When the participant who did not complete the study as planned was removed from the analysis, mean adherence increased to $91.0 \pm 8.5 \%$. Physical activity levels also increased from baseline in all participants although the mean increase for the 5 participants did not reach significance (see figure 1).

Pre- and post-intervention HbA1C data, a primary outcome for individuals with type 2 diabetes, was available for 4 participants. The relative improvement was $10.6 \%$ for these participants. The relative improvements were $13.5 \%$ for 6MWT and 3.9\% for BMI for all participants.
Table 2 Pre-and Post-Intervention data and adherence for individuals who completed the study.

\begin{tabular}{|llll|}
\hline & \multicolumn{2}{l|}{$\begin{array}{l}\text { Exercise and Coaching Application } \\
(\mathrm{N}=5,2 \text { female })\end{array}$} \\
\cline { 2 - 4 } & PRE & POST & $\mathrm{P}$ \\
\hline $\begin{array}{l}\text { Activity minutes/ } \\
\text { week }\end{array}$ & $92.0 \pm 46.6$ & $291.4 \pm 231.7$ & 0.13 \\
\hline BMI & $30.2 \pm 2.6$ & $29.0 \pm 2.3$ & 0.53 \\
\hline $\begin{array}{l}\text { 6MWT distance } \\
(\mathrm{M})\end{array}$ & $476.0 \pm 34.3$ & $540.2 \pm 57.65$ & 0.09 \\
\hline $\begin{array}{l}\text { HbA1C } \\
(\mathrm{mmol} / \mathrm{mol})\end{array}$ & $61.5 \pm 2.6$ & $55.0 \pm 5.2$ & 0.10 \\
\hline $\begin{array}{l}\text { Sessions } \\
\text { attempted }(\%)\end{array}$ & $78.1 \pm 27.6$ & & - \\
\hline $\begin{array}{l}\text { Sessions } \\
\text { completed }(\%)\end{array}$ & $77.8 \pm 27.5$ & & - \\
\hline
\end{tabular}

Figure 1 Pre (blue) v post (grey) intervention self-reported physical activity for individual participants

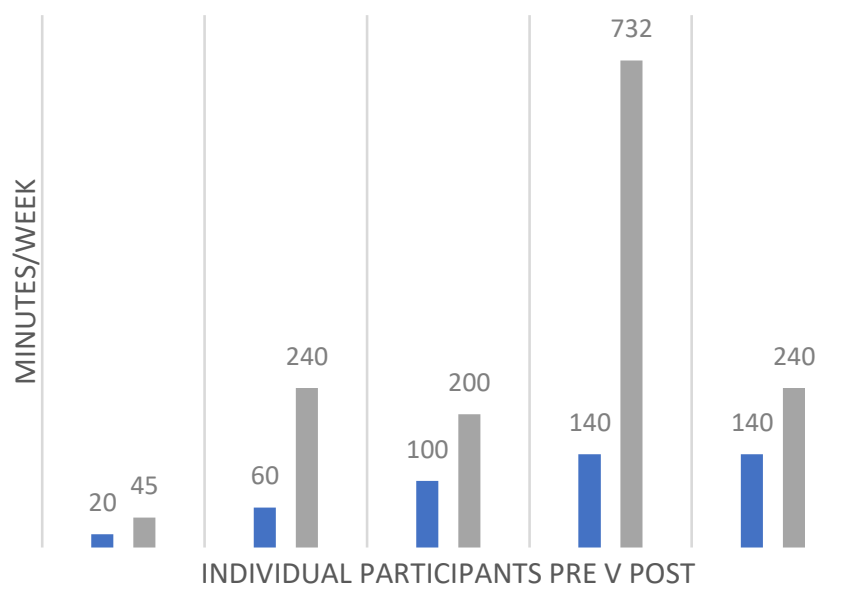

\section{DISCUSSION}

The findings of the study indicate that by combining the simple short bout exercise prescription with the novel smartphone adaptive coaching application used in the study, high adherences and increases in overall physical activity in previously sedentary type 2 diabetics can be produced (see figure 2). Furthermore, the intervention has the potential to improve health related outcomes in the population. Physical activity and minimising sedentary behaviour are fundamental tools used in the successful management of type 2 diabetes. The percent of sessions completed in the group, represent a high adherence for a 16-week exercise intervention of this magnitude. Nonetheless, despite the improvements in physical activity behaviour, 2 individuals in the group did not complete the study although for 1 individual this was due to injury. This highlights some of the challenges associated with yielding high adherences to physical activity in previously sedentary individuals. 
Figure $2 \%$ of exercise sessions completed in weeks 1-7 (blue) and weeks 8-16 (grey) for individual participants

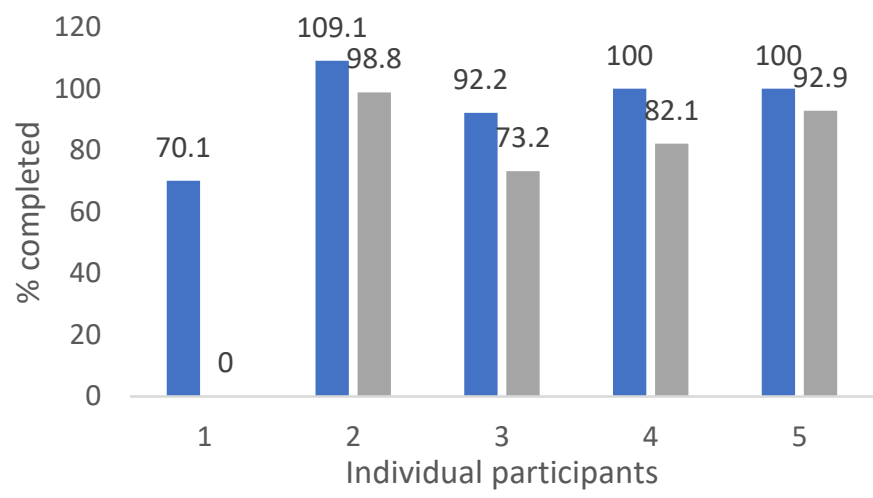

The findings for improvements in $\mathrm{HbA} 1 \mathrm{C}$ in the current study are promising and important clinically, as are the improvements in the other outcomes. Type 2 diabetes is associated with low levels of physical activity and increases in glucose in the bloodstream [7, 8]. Reductions in $\mathrm{HbA} 1 \mathrm{C}$, indicate improvements in glycaemic control in the population. Although the improvements in the measure in in the current study did not reach statistical significance, the sample sizes are small. Increasing the sample sizes may cause the improvements to reach significance. This may also be the case for the other outcomes measured in the study.

The smartphone adaptive coaching application used in the current study presents a novel tool to increase physical activity in previously sedentary populations. Although previous studies have assessed the efficacy of coaching applications in various populations, few, if any, have evaluated the use of a smartphone based adaptive coaching application designed explicitly for a novel 16-week exercise prescription that is aimed at yielding high adherences and improving outcomes. As the study period is only 16 weeks in duration, future work should examine the efficacy of the combination of the progressive exercise intervention and the

\section{REFERENCES}

[1] Booth, F. W., Roberts, C. K. and Laye, M. J. 2012. Lack of Exercise Is a Major Cause of Chronic Diseases. Comprehensive Physiology. 2:1143-1211.

[2] Pedersen BK, Saltin B. Exercise as medicine-evidence for prescribing exercise as therapy in 26 different chronic diseases. Scandinavian journal of medicine \& science in sports. 2015 Dec $1 ; 25(\mathrm{~S} 3): 1-72$.

[3] Byrne H, Caulfield B, De Vito G. Effects of self-directed exercise programmes on individuals with type 2 diabetes mellitus: a systematic review evaluating their effect on $\mathrm{HbAlc}$ and other metabolic outcomes, physical characteristics, cardiorespiratory fitness and functional outcomes. Sports Medicine. $2017 \mathrm{Apr}$ 1;47(4):717-33.

[4] American College of Sports Medicine. ACSM's guidelines for exercise testing and prescription. Lippincott Williams \& Wilkins; 2013 Mar 4.

[5] Pludwinski S, Ahmad F, Wayne N, Ritvo P. Participant experiences in a smartphone-based health coaching intervention for type 2 diabetes: A qualitative inquiry. Journal of telemedicine and telecare. 2016 Apr;22(3):172-8. adaptive coaching application over longer periods. It may be the case that the interventions are less effective over longer periods. Nonetheless, the findings of the current study indicate that the adaptive coaching application is effective in the short term and should be considered in clinical settings in previously sedentary individuals with type 2 diabetes. Likewise, similar adaptive coaching applications and exercise prescriptions should also be trialled in other sedentary populations. Smartphone applications present a major opportunity to clinicians for altering behaviours to improve health outcomes in a wide number of populations.

Future work should increase the sample size to allow for the effects of the interventions seen in the current sample to be confirmed. Furthermore, a larger sample size would allow a more accurate evaluation of the coaching application, regarding its effects on adherence and overall physical activity to be made. An evaluation of the efficacy of the intervention in comparison to just providing the exercise intervention to participants would also allow the effectiveness of the application independently to be made.

In conclusion, the findings of the current study indicate that the smartphone adaptive coaching application designed to be used with a simple novel exercise prescription is a feasible and promising intervention to use in the population. It may have the potential to yield high adherences, increase overall physical activity and improve health related outcome measures in individuals with type 2 diabetes over a 16-week period. Future work is needed to confirm the efficacy of this application and similar adaptive coaching applications with larger sample sizes, over longer periods with longer exercise programmes and in different populations. It may be the case that smartphone adaptive coaching applications in conjunction with specific exercise prescriptions that are also designed to boost adherence and improve health related outcomes bring about the greatest improvements in compliance and health status.

[6] Konrad A, Bellotti V, Crenshaw N, Tucker S, Nelson L, Du H, Pirolli P, Whittaker S. Finding the adaptive sweet spot: Balancing compliance and achievement in automated stress reduction. In Proceedings of the 33rd Annual ACM Conference on Human Factors in Computing Systems 2015 Apr 18 (pp. 3829-3838). ACM.

[7] Helmrich SP, Ragland DR, Leung RW, et al. Physical activity and reduced occurrence of non-insulin-dependent diabetes mellitus. $\mathrm{N}$ Engl J Med. 1991;325(3):147-52.

[8] Snowling NJ, Hopkins WG. Effects of different modes of exercise training on glucose control and risk factors for complications in type 2 diabetic patients: a meta-analysis. Diabetes Care. 2006;29(11):2518-27. 